\title{
Substance Index for Volume 66
}

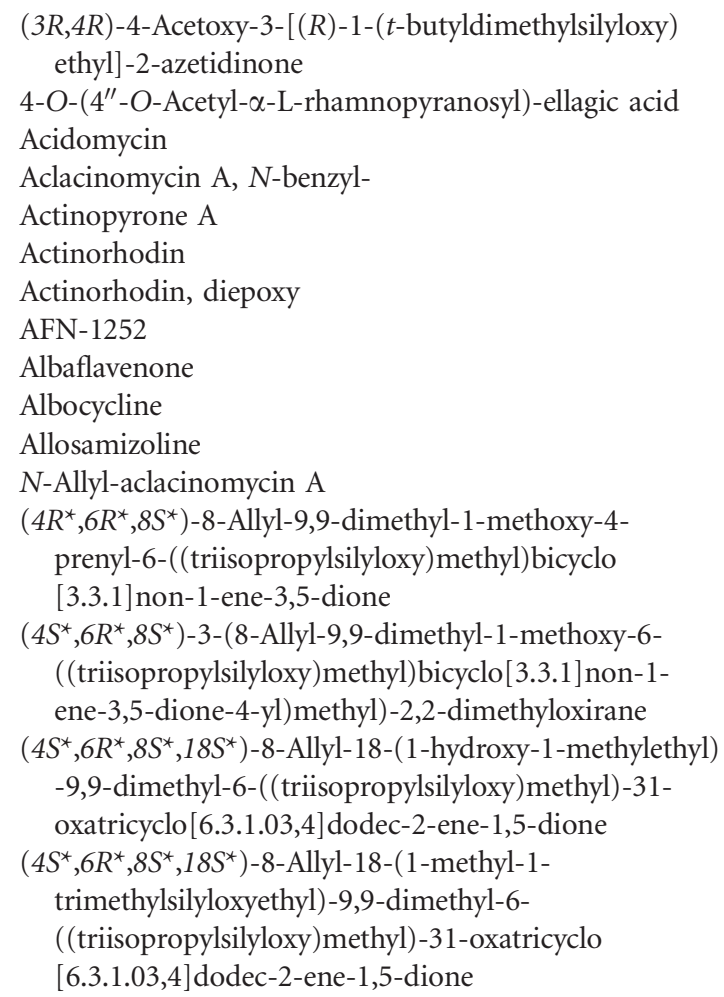

161

123

371

165

123

387

295

571

387

303

123

165

141
A26771B 123

AB3217-A 123

Bedaquiline 571

4-O-Benzoyl-2,3,6-trideoxy-3-C-methyl-3- 131

trifluoroacetamido- $\alpha, \beta$-L-lyxo-hexopyranosyl acetate

4-O-Benzyl-2,3,6-trideoxy-3-C-methyl-3-azido- $\alpha, \beta$-L-lyxo- 131 hexopyranosyl acetate

4-O-Benzyl-2,3,6-trideoxy-3-C-methyl-3-

trifluoroacetamido- $\alpha, \beta$-L-lyxo-hexopyranosyl acetate

$(S, Z)$-3-Benzylidene-6-isopropylpiperazine-2,5-dione 31

(S, Z)-3-Benzylidene-6-methylpiperazine-2,5-dione 31

3-Benzylpiperazine-6-(4-methoxybenzylidene)-2,5-dione 31

Bhimamycin F, H, I $\quad 719$

Biapenem $\quad 571$

Biemodin 491

Brartemicin analogs $\quad 531$

Brilacidin 571

(3S)-4-Bromo-2,3-dimethylbut-1-ene 147

(2E,5R)-1-tert-Butyldiphenylsilyloxy-5- 155

methanesulfonyloxy-2-methylhex-2-ene

BAL30072 571

BC-3781 571

BE-14106 431

BE-52440A 107, 123

BE-54238B 107

BE-5423B 123

Cabanillasin 617

$\begin{array}{ll}\text { Cadazolid } & 371,571\end{array}$

Calbistrin A 123

Caloporoside, deacetyl- $\quad 123$

Caprazene, $5^{\prime \prime}-N$-t-butoxycarbonyl $\quad 171$

Caprazene 4-butylanilide $\quad 171$

Caprazene tetradecylamide $\quad 171$

Caprazene tridecyl ester $\quad 171$

Carbapen-2-em-3-carboxylic acid 401

Carbapenems, olivanic acid-type 401

Carbomycin B 123

Cefazolin $\quad 511$

Cefmetazole $\quad 97$

Cefotiam 511

$\begin{array}{lr}\text { Cefoxitin } & 97\end{array}$

Cefozopran 107

Ceftaroline $\quad 571$

Ceftazidime 571, 593

Ceftolozane $\quad 571$

Cephabacins 401

Cephalosporins 401

Cephamycin 401

Chloramlincomycin $\quad 371$

$\begin{array}{lr}\text { Chloramphenicol } & 97\end{array}$

[5-(4-Chlorophenyl)-5-hydroxy-3-(trifluoromethyl)- 371 4,5-dihydro-1H-pyrazol-1-yl] (m-tolyl)methanone

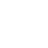

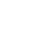

1

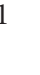

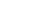

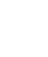

1


Chymostatin A, B, C 123

Cilastatin 571

Ciprofloxacin 593

Citreorosein $\quad 219$

5S Clavams 401

Clavulanic acid 401, 411

Clindamycin 97, 511

Cochleamycin A 107, 123

epi-Cochlioquinone A 123

Coelichelin $\quad 387$

Coelimycin P1 387

$\begin{array}{ll}\text { Colistin } & 705\end{array}$

Coriolin 123

Cuevaene A 563

Curvalarol A, B $\quad 735$

Curvularone A 287

Cyclomenol A $\quad 431$

Cyclophellitol 123

Cylindrol $\mathrm{A}_{5}$

Cystacyclin $\quad 743$

CB-182,804 371

CBR-2092 371

CG400549 571

Dalbavancin $\quad 571$

Danofloxacin $\quad 629$

Delafloxacin $\quad 571$

Delamanid $\quad 571$

Desferrioxamine 387

$\left(4 S^{\star}, 6 R^{\star}, 8 S^{\star}, 18 S^{\star}\right)$-2,8-Diallyl-18-(1-hydroxy-1- 141

methylethyl)-6-(1-hydroxy-2-methylpropyl)-9,9-

dimethyl-31-oxatricyclo[6.3.1.03,4] dodec-2-ene1,5-dione

$\left(4 S^{\star}, 6 R^{\star}, 8 S^{\star}, 18 S^{\star}\right)-2,8$-Diallyl-18-(1-hydroxy-1methylethyl)-6-hydroxymethyl-9,9-dimethyl-31oxatricyclo[6.3.1.03,4] dodec-2-ene-1,5-dione

$\left(4 S^{\star}, 6 R^{\star}, 8 S^{\star}, 18 S^{*}\right)-2,8$-Diallyl-18-(1-hydroxy-1methylethyl)-6-isobutyryl-9,9-dimethyl-31-oxatricyclo[6.3.1.03,4] dodec-2-ene-1,5-dione

$\left(4 S^{\star}, 6 R^{\star}, 8 S^{\star}, 18 S^{\star}\right)-2,8$-Diallyl-18-(1-hydroxy-1methylethyl)-9,9-dimethyl-31-oxatricyclo[6.3.1.03,4] dodec-2-ene-1,5-dione-6-carbaldehyde

$\left(4 S^{\star}, 6 R^{\star}, 8 S^{\star}, 18 S^{\star}\right)-2,8$-Diallyl-18-(1-methyl-1trimethylsilyloxyethyl)-9,9-dimethyl-6((triisopropylsilyloxy)methyl)-31-oxatricyclo [6.3.1.03,4] dodec-2-ene-1,5-dione

(3S)-2,3-Dimethyl-4-(p-toluenesulfonyl)oxybut-1-ene

Dinapinone AB1, AB2, AC1, AC2, AD1, AD2, AE1, AE2

Diorcinol B, C, D, E

(1S,2R,5R)-2-((1,3-Dioxolan-2-yl)methyl)-1-((Z)-1methoxy-2-(ethoxycarbonyl)ethenyl)-2,4,5trimethylcyclohex-3-en-1-ol

(1S,2R,5R)-2-((1,3-Dioxolan-2-yl)methyl)-1 ([2-(ethoxycarbonyl)ethynyl)-2,4,5-trimethylcyclohex3 -en-1-ol

(2R)-2-((1,3-Dioxolan-2-yl)methyl)-2-methylbut3 -en-1-ol

(2R)-2-((1,3-Dioxolan-2-yl)methyl)-2-methylbut-3-enal

(2R,5R)-2-((1,3-Dioxolan-2-yl)methyl)-2,4,5trimethylcycohex-3-en-1-one
(3R,6R)-3-[(1,3-Dioxolan-2-yl)methyl]-3,6,7-

trimethylocta-1,7-dien-4-one

(5R,6R,9R)-6-((1,3-Dioxolan-2-yl)methyl)-6,8,9-

trimethyl-1-oxaspiro[4.5] deca-3,7-dien-2-one

(5S,6R,9R)-6-((1,3-Dioxolan-2-yl)methyl)-4-

methoxy-6,8,9-trimethyl-1-oxaspiro[4.5]deca-3,7dien-2-one

DS-8587

Ebelactone A

Elaiomycins K, L

Elaiophylin

Emodin

Epothilone O

Eravacycline

Erbstatin

$(11 R, 12 S)$-Erythro-mefloquine, $(-)-,(+)-$

Erythromycin A

2-(Ethoxycarbonyl)propanoic acid

107, 123

Exeporfinium chloride

ES-242-4, -5

Falcitidin

Fidaxomicin

Finafloxacin

571

Fluconazole

651

Fosfomycin

625, 709

Garsubellin A

141

Geldanamycin, 19-S-methyl

499

Gentamicin

511

Geosmin

Germicidin

387

123

Gualamycin

571

GSK1322322

Herbimycin A

Hibarimicinone

107, 123

Hirsutene

123

Hitachimycin

431

Hopene

387

1-Hydroxy-4-methoxy-2-naphthoic acid

IMC0301B

Imipenem

97, 511, 571

Incednam

Incednine

Indanostatin

431, 691

Indisocin

731

123

543

Intervenolin

387

Isorenieratene

Isoretronecanol

JBIR-130, -131, 132

Kalafungin

107,123

Kanamycin A

107, 123

Kanamycin B, C

KB425796-A, -C 
KB425796-B, -D, -E, -F, -G, -H, -I, -J, -K

K1115 B1 $\alpha, B 1 \beta$

KD16-U1

KPI-10 (WQ-3813)

Lactonamycin

107, 123

Lagunamycin

Langkocycline A1, A2, A3, B1, B2

Leucomycin A3

Levofloxacin

Lincomycin derivatives

107, 123

511, 709

195

Lincophenicol

Linearolide A, B

Linezolid

Linfuranone A

Linieodolide A, B $\quad 89$

Lorneic acid C, D 347

Luminacin C1, C2 123

Lymphostin 123

LCB01-0371 571

LFF-571 571

LL-37 571

LL-Z1640-2 123

LTX-109 571

Macplocimine A 443

Macrocyclic engelhardione analogs $\quad 319$

Macrolactin X, Y, Z $\quad 89$

Maniwamycin A, B $\quad 123$

MB12, 13

MBJ-0009, -0010

MBJ-0011, -0012, -0013 299

MBJ-0020 563

MBJ-0038, -0039, -0040 727

MBX 1066, 1090, 1113, 1128, 1162

$\begin{array}{ll}\text { Medermycin } & 107,123\end{array}$

Methicillin 517

(3Z, 6Z)-3-(4-Methoxybenzylidene)-6- 31

(2-methylpropylidene)piperazine-2,5-dione

per-6-(4-Methoxylbenzyl)-amino-6-deoxy- $\beta$ cyclodextrin

2-Methoxynaphthalene-1,4-dione

Methyl 2,3,6-trideoxy-3-C-methyl-3-trifluoroacetamido$\beta$-L-lyxo-hexopyranoside

Methyl 2,3-di-O-(tert-butyldimethylsilyl)-6-deoxy-4-O((2R)-2-((1,3-dioxolan-2-yl)methyl)-2-methyl-3butenoyl)- $\alpha$-D-glucopyranoside

Methyl 2,3-di-O-(tert-butyldimethylsilyl)-6-deoxy-4-O((2R)-2-ethoxycarbonyl-2-formylmethylpropanoyl)$\alpha$-D-glucopyranoside

Methyl 2,3-di-O-(tert-butyldimethylsilyl)-6-deoxy-4-O((2R)-2-ethoxycarbonyl-2-methylpent-4-enoyl)$\alpha$-D-glucopyranoside

Methyl 2,3-di-O-(tert-butyldimethylsilyl)-6-deoxy-4-O((2R)-2-(1,3-dioxolan-2-yl)methyl-2-(ethoxycarbonyl) propanoyl $)-\alpha$-D-glucopyranoside

Methyl 2,3-di-O-(tert-butyldimethylsilyl)-6-deoxy-4-O((2R)-2-(1,3-dioxolan-2-yl)methyl-2-formylpropanoyl)$\alpha$-D-glucopyranoside

Methyl 2,3-di-O-(tert-butyldimethylsilyl)-6-deoxy-4-O(2-(ethoxycarbonyl)propanoyl)- $\alpha$-D-glucopyranoside
Methyl 2-hydroxy-3-(4-O-benzoyl-2,3,6-trideoxy-3-C-

131 methyl-3-trifluoroacetamido- $\beta$-L-lyxohexopyranosyl)-6-(prop-2-ene-1-yloxy)benzoate

Methyl 2-hydroxy-3-(4-O-benzyl-2,3,6-trideoxy-3-Cmethyl-3-azido- $\beta$-L-lyxo-hexopyranosyl)-6(prop-2-ene-1-yloxy)benzoate

Methyl 2-hydroxy-3-(4-O-benzyl-2,3,6-trideoxy-3-Cmethyl-3-trifluoroacetamido- $\beta$-L-lyxo-hexopyranosyl)6-(prop-2-ene-1-yloxy)benzoate

$N$-Methylindisocin 123

O-Methyl methyl 4,6-O-benzylidene-2,3-dideoxy- $\alpha$ - $\quad 131$ D-erythro-hexopyranosid-3-ulose oxime

Methyl 3-O-(tert-butyldimethylsilyl)-6-deoxy- $\alpha$-Dglucopyranoside

Methyl 4,6-O-benzylidene-2,3-dideoxy-3( $N$-methoxytrifluoroacetamido)-3-C-methyl- $\alpha$-D-ribohexo-pyranoside

Methyl 4,6-O-benzylidene-2,3-dideoxy-3-C-methyl-3(methoxyamino)- $\alpha$-D-ribo-hexopyranoside

Methyl 4,6-O-benzylidene-2,3-dideoxy-3-C-methyl-3trifluoroacetamido- $\alpha$-D-ribo-hexopyranoside

Methyl 4-O-benzoyl-2,3,6-trideoxy-3-C-methyl-3trifluoroacetamido- $\alpha$-D-erythro-hex-5-enopyranoside

Methyl 4-O-benzoyl-2,3,6-trideoxy-3-C-methyl-3trifluoroacetamido- $\alpha$-D-ribo-hexopyranoside (epi-8)

Methyl 4-O-benzoyl-2,3,6-trideoxy-3-C-methyl-3trifluoroacetamido- $\beta$-L-lyxo-hexopyranoside

Methyl 4-O-benzoyl-6-bromo-2,3,6-trideoxy-3-Cmethyl-3-trifluoroacetamido- $\alpha$-D-ribo-hexopyranoside

Methyl 4-O-benzyl-2,3,6-trideoxy-3-C-methyl-3-amino$\beta$-L-lyxo-hexopyranoside

Methyl 4-O-benzyl-2,3,6-trideoxy-3-C-methyl-3-azido$\beta$-L-lyxo-hexopyranoside

Methyl 4-O-benzyl-2,3,6-trideoxy-3-C-methyl-3dimethylamino- $\beta$-L-lyxo-hexopyranoside

Methyl 4-O-benzyl-2,3,6-trideoxy-3-C-methyl-3trifluoroacetamido- $\beta$-L-lyxo-hexopyranoside

Methylenomycin

Methylfurans

$(2 Z, 4 E, 6 E, 8 E, 10 R, 11 S, 12 Z)-1-((2 E, 4 E, 6 E)-5$-Methylhepta4,6-diene)amide-10,11-bis(triethylsilyloxy)-13-iodo-2methoxy-4,10,16-trimethyltrideca-2,4,6,8,12-pentaene

$(2 Z, 4 E, 6 E, 8 E, 10 R, 11 S, 12 Z, 14 E, 16 E, 18 E)-1-((2 E, 4 E, 6 E)-$ 5-Methylhepta-4,6-diene)amide-10,11bis(triethylsilyloxy)-13-iodo-2-methoxy-4,10,16trimethylnonadeca-2,4,6,8,12,14,16-heptaene

(1E,3E,5E)-3-Methylhex-1,3,5-trien-1-tributylstannane

Metronidazole

Micromonosporin A $\quad 431$

Migracin A, B $\quad 225$

Minocycline $\quad 511$

Monamphilectine A 401

Monapinone A, B 179

Monobactams, sulfazecin-type 401

Monodeacetylphomoxanthon B 231

Multhiomycin $\quad 599$

MCB-3837 371

MK-7655 571

ML-449

MRX-I 571

MS-444 123 
Nacardicyclins

(S)-Nadifloxacin

Nagstatin

Nanaomycin A, D, E

Napyradiomycin A1

107, 123

Neamine

Neihumicin, methoxy

Nemonoxacin

Neopyrrolomycin

Nidulalin A

Nocardicin A

NAB739

NAI-802

NVB302

Oleandomycin

Olivomycin A

Omadacycline

Orbifloxacin

Oritavancin

Ozenoxacin

OM-173 $\alpha \mathrm{E}$

Pachystermine A

Paecilin C

Penicillin G

Penicillins

Penicillixanthone A

Perchlozone

Phenethylamine derivatives

1,1' [1,2-Phenylenediylbis(nitrilomethylidine)] bis-(2-naphthol)

1,1' [1,2-Phenylenediylbis(nitrilomethylidine)] bis-2-naphtholatocopper(II)

$O$-Phenylthiomethyltriazolyloxime

Philipimycin

Piperacillin

Piprofloxacin

Platencin

Platensimycin

Plazomicin

Pluramycin A

Polymyxin E

Posizolid

Prodiginine

Pyralomicin 1c, 2c

Pyrenomycin, benzo

Pyridomycin

Pyrizinostatin

Pyrrolizilactone

PA-824

PC-3

PF1092A, B, C

PF1163A, B

PJS

Quinolactacin B

$\mathrm{O}$-(3-Quinolyltriazolylmethyl)oxime

Radezolid

Radianthin, 4-hydroxy

107,123

107,123
Reveromycin derivatives

Riboflavin, 2(4)-imino-4(2)-amino-2,4-dideoxy

Riboflavin, 8-demethyl-8-amino

Rifampicin

Rifamycin W

Roseoflavin

Rosmarinecine

RPX7009

$\mathrm{Rx}-2102$

Saccharocin

107,123

Saccharosporone A, B, C 305

Salinilactam A $\quad 431$

Sarecycline $\quad 571$

Sceliphrolactam 431

Secalonic acid B, D $\quad 219$

Sideroxylonal B, C $\quad 123$

Sinefungin $\quad 371$

Solithromycin $\quad 571$

Sparsophenicol $\quad 371$

Spirohexaline $\quad 37$

Spliceostatin B $\quad 555$

Streptorubin B 387

$\begin{array}{ll}\text { Sulbactam } & 705\end{array}$

Sulfomycin I $\quad 599$

Surotomycin $\quad 571$

Sutezolid 571

S54832 A-1 599

SCB1 387

SQ109 571

$\begin{array}{ll}\text { Tabtoxin } & 401\end{array}$

Tazobactam $\quad 571$

Tedizolid $\quad 571$

Tedizolid phosphate $\quad 571$

Teicoplanin 273, 511

Teicoplanin A2-1, A2-2, A2-3, A2-4, 641 A2-5, A3-1

Terpestacin 123

Tetracycline 107, 123

5-(1,5,6,7-Tetrahydro-6,7-dioxo-8-D-ribityllumazin- 371 5-yl-)pentane 1-phosphate

6,8,5'6'-Tetrahydroxy-3'-methylflavone 219

Tetrodecamycin 107, 123

Thiazomycin 107, 123, 401, 599

Thiocillin I, II 599

Thiostrepton A $\quad 599$

THN/flaviolin $\quad 387$

Tigecycline $\quad 705$

Trehalosamine $\quad 123$

Trehangelin A, B, C 311

epi-Trichosetin $\quad 549$

Trichostatin D 123

3S, 22R, 26-Trihydroxy-8, 24E-euphadien-11-one 679

Tsugicoline A 43

Tubelactomicin A 107, 123

Tylopilusin C 355

Tylosin 107, 123

TD-1792 371

TMC-66, -264 123 
UCE 6

UK-80882

Validamine

123

Valienamine

Vancomycin

Versipelostatin

Versipelostatin F

Vicenistatin

Vinaxanthone

Viridenomycin

Viridicatumtoxin

Voriconazole

Waldiomycin

Warkmycin

459

669
Warkmycin, 4-O-deacetyl-

Wortmannin B

669

491

Xanthocillin X 123

Xanthocillin X dimethyl ether 123

Xanthone derivatives 453

XR334

XR774

31

YCM1008A

123

YM182029

123

YM-30059

123

Zabofloxacin

571

0002-04-KK

663

0026-59-KK

663

03219A

327 